\title{
Thymidine phosphorylase expression and prognosis in colorectal cancer treated with 5-fluorouracil-based chemotherapy: A meta-analysis
}

\author{
JIA CHE $^{1-3^{*}}$, LUN PAN $^{4 *}$, XIANGLING YANG ${ }^{1}$, ZHITING LIU $^{5}$, LANLAN HUANG ${ }^{1-3}$, \\ CHUANGYU WEN ${ }^{1-3}$, AIHUA LIN ${ }^{4}$ and HUANLIANG LIU ${ }^{1-3}$
}

\begin{abstract}
${ }^{1}$ Guangdong Provincial Key Laboratory of Colorectal and Pelvic Floor Diseases, Guangdong Institute of Gastroenterology and
${ }^{2}$ Department of Clinical Laboratory, The Sixth Affiliated Hospital, Sun Yat-sen University; ${ }^{3}$ Key Laboratory of

Tropical Disease Control of Ministry of Education, Sun Yat-sen University, Guangzhou, Guangdong 510655;

${ }^{4}$ Department of Medical Statistics and Epidemiology, School of Public Health, Sun Yat-sen University,

Guangzhou, Guangdong 510000; ${ }^{5}$ Institute of Nutrition and Food Safety, Guangdong Provincial

Center for Disease Control and Prevention, Guangzhou, Guangdong 511430, P.R. China
\end{abstract}

Received January 14, 2017; Accepted August 17, 2017

DOI: $10.3892 / \mathrm{mco} .2017 .1436$

\begin{abstract}
In the past decades, various studies have suggested a possible link between thymidine phosphorylase (TP) level and colorectal cancer (CRC) treated with 5-fluorouracil (5-FU)-based chemotherapy; however, they have arrived at inconsistent results. Therefore, the present meta-analysis aimed to disclose a more comprehensive evaluation of this relationship. PubMed, the Cochrane Library, Ovid MEDLINE, Embase and China National Knowledge Infrastructure were systematically searched for studies that evaluated the prognostic value of TP in CRC. Stata 12.0 software was used to test the heterogeneity and evaluate the overall test performance. A total of 15 studies, including 1,225 patients, were included. The summary estimates of TP for CRC treated with 5-FU-based chemotherapy indicated a moderately positive prognosis with a hazard ratio $(\mathrm{HR})$ of $0.76(\mathrm{P}=0.031)$ for overall survival and a HR of $0.711(\mathrm{P}=0.022)$ for relapse-free survival. On the basis of the present meta-analysis, TP could be promising and
\end{abstract}

Correspondence to: Professor Huanliang Liu, Guangdong Provincial Key Laboratory of Colorectal and Pelvic Floor Diseases, Guangdong Institute of Gastroenterology, The Sixth Affiliated Hospital, Sun Yat-sen University, 26 Yuancun Er Heng Road, Guangzhou, Guangdong 510655, P.R. China

E-mail: liuhuanl@mail.sysu.edu.cn

Professor Aihua Lin, Department of Medical Statistics and Epidemiology, School of Public Health, Sun Yat-sen University, 74 Zhongshan Er Road, Guangzhou, Guangdong 510000, P.R. China E-mail: linaihua@mail.sysu.edu.cn

*Contributed equally

Key words: 5-fluorouracil, thymidine phosphorylase, colorectal cancer, chemotherapy, meta-analysis meaningful in the prognosis of CRC treated with 5-FU-based chemotherapy.

\section{Introduction}

Colorectal cancer (CRC) is one of the most common human malignant tumors worldwide and morbidity associated with CRC is increasing annually (1). It has been reported that CRC was the third most commonly diagnosed cancer in males and the second in females, with $\sim 1.4$ million cases and 693,900 mortalities occurring in 2015 (2). To date, 5-fluorouracil (5-FU) is widely used as a primary chemotherapeutic agent and constitutes the fundamental basis of chemotherapy treatment for patients with CRC since it was introduced in 1957 (3-6). Although targeted epidermal growth factor receptor monoclonal antibodies, namely cetuximab and panitumumab, have been introduced and have been used for many patients with CRC and benefits are achieved, 5-FU is still a basic chemotherapy for CRC clinical treatment (7). Furthermore, several agents, such as 5-FU plus leucovorin (LV) or infusional 5-FU plus LV and oxaliplatin, have been established as the generalized regimen for the treatment of patients with CRC (8). For example, according to the guidelines of the National Comprehensive Cancer Network, FOLFOX6 chemotherapy including continuous infusion of 5-FU combined with oxaliplatin and calcium folinate has become the standard chemotherapy regimen for postoperative patients with CRC $(9,10)$. In addition, oral forms of 5-FU-related drugs, such as capecitabine or tegafur plus uracil and doxifluridine (5'-DFUR), have also been developed for convenient administration and have been widely used in patients with CRC (11-13). However, the clinical effectiveness of 5-FU-based chemotherapy differs among patients (14). It is important to select 5-FU-based chemotherapy so that each patient may benefit and experience the least harmful side effects. To predict the clinical efficacy of 5-FU-based chemotherapy in CRC patients, it is essential to define a predictive biomarker associated with 5-FU treatment. 
Thymidine phosphorylase (TP) is an important metabolizing enzyme that catalyzes the conversion of 5-FU to its more active nucleoside form, 5-fluoro-2'-deoxyuridine, representing one of the main pathways through which this drug exerts its cytotoxic effect (15). One of the roles of TP is controlling the intracellular levels of thymidine, which at higher concentrations becomes toxic to cells and causes replication errors in DNA (16). Research has demonstrated that the levels of TP are higher in tumors compared with normal tissues in a wide range of solid tumors (15,17-20). The expression of TP may be correlated with the efficacy of 5-FU-based chemotherapy (21). However, in cancer development, it has been reported that TP functions as the molecule platelet-derived endothelial cell growth factor in cells and exhibits angiogenic properties in tumors $(22,23)$. In 2009, a study by Bronckaers et al (15) reported that TP had a dual role in cancer development. TP may prevent apoptosis and induce angiogenesis to promote tumor growth and metastasis, which is the targeted function of TP inhibitors; however, TP is also indispensable for the activation of the extensively used 5-FU prodrugs, such as doxifluridine and capecitabine (15). As it has been demonstrated that TP has a complicated role in CRC development and 5-FU treatment, whether TP may predict the prognosis of patients with CRC treated with 5-FU-based chemotherapy remains uncertain. Various studies $(4,5,24,25)$ have investigated the association between the levels of TP and survival in CRC patients; however, a certain conclusion regarding this has not been drawn. Although the majority have reported poorer overall survival (OS) and progression-free survival (PFS) in patients with tumors expressing high TP levels, there are also reports that have demonstrated no association between them, resulting in greatly different estimates of the prognostic value of TP expression between studies (26-29). The ability to use TP expression to predict the response of patients with CRC to 5-FU-based chemotherapy thus remains controversial.

The aim of the present study was to evaluate the scientific evidence for the effect of TP expression in patients with CRC treated with 5-FU-based chemotherapy by using a standard meta-analysis of data from published studies.

\section{Data collection methods}

Search strategy. Searches were conducted on Wiley Online Library (onlinelibrary.wiley.com), Scopus (scopus. com/home.uri), PubMed (ncbi.nlm.nih.gov/pubmed), the Web of Science (webofknowledge.com), the Cochrane library (cochranelibrary.com), Ovid MEDLINE (hsl.lib.umn. edu/biomed/help/ovid-medline), SinoMed (sinomed.ac.cn) and China National Knowledge Infrastructure (CNKI; cnki. net) without language limitation. The last search update was April 28, 2015. The search strategy predominantly included terms suggestive of four factors: i) TP (i.e., 'thymidine phosphorylase', 'platelet-derived endothelial cell growth factor' and 'PD-ECGF'); ii) 5-FU (i.e., '5-fluorouracil', 'adrucil', 'carac', 'efudex', 'efudix', '5-fluoro-1H, 3H-pyrimidine-2, 4-dione' and '5-fluoropyrimidines'); iii) colorectal (i.e., 'colon', 'rectal', 'colorectal' and 'rectum'); and iv) cancer (i.e., 'cancer', 'carcinoma', 'neoplasm', 'tumor' and 'malignant'). Article types were restricted to clinical trials or randomized controlled trials in humans. The reference lists of primary studies and previous meta-analyses were scrutinized for additional publications.
Inclusion and exclusion criteria. The potential trials were screened according to the following criteria: i) Patients had a diagnosis of CRC; ii) all patients received 5-FU-based chemotherapy; iii) the studies reported one or more indicators, including objective response rate (ORR), PFS, disease-free survival (DFS), relapse-free survival (RFS) and OS, to compare the prognosis of patients stratified by TP expression. Studies providing information on survival were included, while studies without survival analysis, investigating response rates only were excluded; iv) the results were part of the original analysis; v) when results reported by the same author were acquired from the same patient population in more than one publication, only the study involving the highest number of patients was included; and vi) retrospective, prospective or randomized controlled trials were included. Trials evaluating progression with time to tumor progression, which was defined as time from the initiation date of 5-FU-based chemotherapy to the first radiographic evidence of disease progression or mortality, were also included. Trials lacking complete data that were still in progress and without full text articles online were excluded. The present study attempted to obtain the data with the longest follow-up when reports overlapped or were repeated.

Data extraction and definitions. Data extracted included the first author, publication year, study type, chemotherapy regimen, lesions tested, TP evaluation method, study size, high TP level and percentage of patients with high TP expression. For clinical outcome, the number of responders were collected for calculating odds ratio (OR) and $95 \%$ estimation intervals for ORR. Hazard ratios (HRs) and 95\% confidence intervals (CI) were also extracted for OS, PFS, DFS and RFS. If a separate HR was not provided, the HR and its variance were estimated from the published survival curves using previously described methods and models $(30,31)$. Adjusted HRs and estimation intervals were also collected when reported. Objective response included complete response and partial response, and non-response consisted of stable disease and progressive disease, according to the Response Evaluation Criteria in Solid Tumors (32) or World Health Organization criteria (33). PFS was defined as the time from the initiation date of 5-FU-based therapy to the first evidence of disease progression or mortality from any cause. OS was defined as the time from the initiation date of 5-FU-based therapy to mortality from any cause. DFS was defined as the length of time after being treated with 5-FU-based therapy during which no disease was found. RFS was defined as the length of time after being treated with 5-FU-based therapy during which patients survived without any signs or symptoms of CRC. All data were extracted by two independent investigators. Discussions were used to reach an agreement if discrepancies existed.

Statistical analysis. The required information was extracted by two independent reviewers using pre-determined data extraction forms. The data were analyzed using Stata 12.0 software (StataCorp LP, College Station, TX, USA). Heterogeneity across studies was evaluated by the Q test and were quantified by the $\mathrm{I}^{2}$ test. If the tests of heterogeneity were significant $(\mathrm{P}<0.05)$, the effect sizes were calculated with the random effects model using the DerSimonian-Laird method. Otherwise, the 


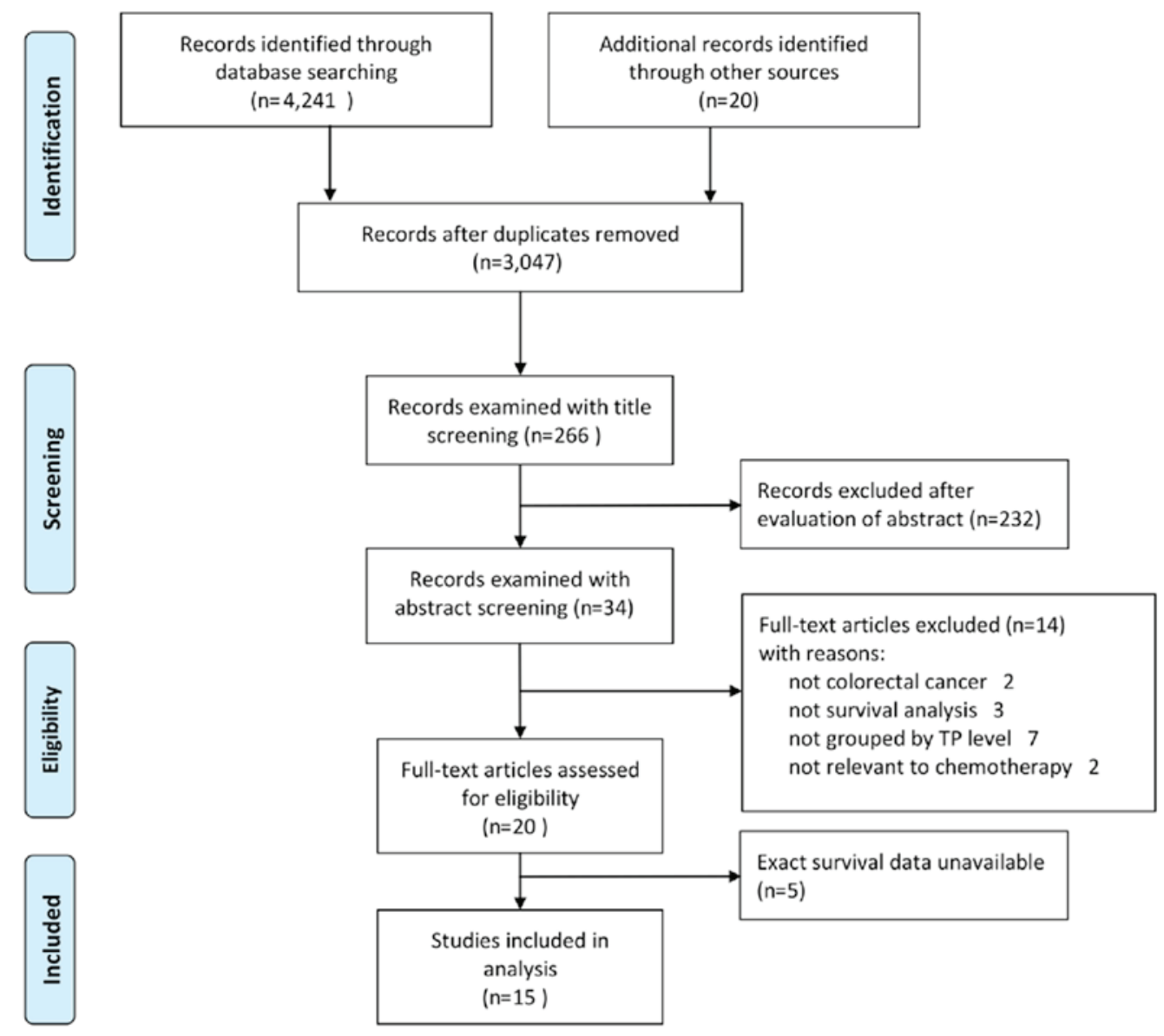

Figure 1. Flow chart of article selection for the present meta-analysis.

fixed-effect model with inverse variance weights was used. A funnel plot and an Egger test were used to assess publication bias. Subgroup analysis was conducted in the different treatment settings and TP detection methods. Sensitivity analysis was used to test the stability when large heterogeneity was presented. All P-values reported were two-sided. Publication biases were assessed by the Egger's test $(\mathrm{P}<0.05$ indicated an existing publication bias) and were reflected by the symmetry of the funnel plot on the natural logarithm of RRs or HRs (34).

\section{Results}

Study selection and characteristics. The search strategy identified 3,047 potentially relevant articles (398 from the Web of Science, 1,609 from the Wiley Online Library, 669 from Scopus, 237 from PubMed, 3 from Ovid MEDLINE, 17 from the Cochrane Library, 22 from SinoMed and 73 from CNKI). Following review of the titles, 266 of these studies were included. Subsequently, a total of 232 studies were excluded following abstract review. Among the 34 studies remaining, two were not relevant to CRC (studying TP in gastric cancer and hepatocellular carcinoma) and three were not survival analyses (comparing the TP level in different tissue or other research). There were seven studies in which the outcomes were not compared at different TP levels and two studies that were not relevant to 5-FU chemotherapy. Thus, 14 articles were excluded and 20 studies were eligible for data extraction. However, five of these 20 studies did not offer eligible data and it was no possible to obtain exact survival information from these five articles. After completing the selection process, data from a total of 15 studies $(4,5,24-29,35-41)$ involving 1225 patients (Fig. 1) were systematically analyzed.

Main characteristics for individual studies were summarized in Table I, including nationality, study type, 5-FU-based drugs and chemotherapy regimens. Of the 15 studies, $9(4,24,25,27,36-39,41)$ were conducted in Asia and seven were from Japan. A total of $10(4,5,25,26,28,35,37-39,41)$ were retrospective studies, one (29) was a prospective study and the other four $(24,27,35,40)$ were not stated. Although the chemotherapy regimen varied in all 15 articles, they all used 5-FU-based drugs. Additionally, TP expression for individual studies were summarized in Table II. Treatment setting was separated into two kinds, primary and metastatic tumors, according to their different chemotherapy regimens. Of the studies, six articles studied primary tumors, seven studied metastatic tumors and two studied both. In terms of follow-up period, 10 articles reported the median follow-up period while another five articles did not. There were 13 studies $(4,5,24-29,35-38,41)$ that stated the lesion tested. Among them, one study was tested on metastatic cancer tissue, 10 on primary tissue and two on both. There were two TP evaluation methods used among these studies, immunohistochemistry (IHC) and quantitative polymerase chain reaction (qPCR).

Assessment of study quality. To conduct the quality assessments for the 15 studies, the Newcastle-Ottawa Scale (NOS) 
Table I. Summary of main characteristics for individual studies.

\begin{tabular}{|c|c|c|c|c|c|}
\hline $\begin{array}{l}\text { Author/(Refs.), } \\
\text { year }\end{array}$ & Nationality & $\begin{array}{l}\text { Study } \\
\text { type }\end{array}$ & $\begin{array}{l}\text { 5-FU-based } \\
\text { drugs }\end{array}$ & $\begin{array}{l}\text { Chemotherapy } \\
\text { regimen }\end{array}$ & $\begin{array}{l}\text { Quality } \\
\text { score }\end{array}$ \\
\hline Ahn et al (24), 2005 & Korea & NS & $5-\mathrm{FU}$ & FOLFIRI or FOLFOX & 7 \\
\hline Kataoka et al (25), 2015 & Japan & Retrospective & $5-\mathrm{FU}$ & $\begin{array}{l}\text { FOLFOX + bevacizumab } \\
\text { or FOLFOX + cetuximab }\end{array}$ & 6 \\
\hline Shigeta et al (4), 2014 & Japan & Retrospective & $5-\mathrm{FU}$ & $5-\mathrm{FU}+\mathrm{LV}$ or $\mathrm{UFT}+\mathrm{LV}$ & 6 \\
\hline Ogawa et al (35), 2014 & Japan & NS & $5-\mathrm{FU}$ & S-1 & 7 \\
\hline Donada et al (36), 2011 & Italy & Retrospective & $5-\mathrm{FU}$ & $5-\mathrm{FU}+\mathrm{LV}$ & 6 \\
\hline Petrioli et al (26), 2010 & Italy & Retrospective & $5-\mathrm{FU}$ & $5-\mathrm{FU}$ or $\mathrm{CAP}$ & 5 \\
\hline Lindskog et al (5), 2014 & Swiss & Retrospective & $5-\mathrm{FU}$ & $\begin{array}{l}5-\mathrm{FU}+\mathrm{LV} \text { or } 5-\mathrm{FU}+\mathrm{OX} \text { or } \\
\mathrm{MIFL} \text { or CAP or CAP }+\mathrm{OX} \text { or } \\
\mathrm{CAP}+\mathrm{IRI}\end{array}$ & 6 \\
\hline Yamada et al (27), 2008 & Japan & NS & $5-\mathrm{FU}$ & UFT or UFT + LV & 6 \\
\hline Jensen et al (28), 2008 & Denmark & Retrospective & $5-\mathrm{FU}$ & Mayo & 7 \\
\hline Yanagisawa et al (37), 2007 & Japan & Retrospective & $5-\mathrm{FU}$ & MIFL & 6 \\
\hline Meropol et al (29), 2006 & USA & Prospective & $5-\mathrm{FU}$ & CAP + IRI & 6 \\
\hline Ichikawa et al (38), 2003 & Japan & Retrospective & $5-\mathrm{FU}$ & $\mathrm{UFT}+\mathrm{LV}$ & 7 \\
\hline Tokunaga et al (39), 2002 & Japan & Retrospective & $5-\mathrm{FU}$ & UFT & 7 \\
\hline Metzger et al (40), 1998 & USA & NS & $5-\mathrm{FU}$ & 5-FU/LV & 7 \\
\hline Soong et al (41), 2008 & Singapore & Retrospective & $5-\mathrm{FU}$ & 5-FU/LV & 6 \\
\hline
\end{tabular}

FOLFIRI, 5-FU + leucovorin + irinotecan; FOLFOX, 5-FU + leucovorin + oxaliplatin; UFT, oral tegaful + uracil; S-1, tegafur + gimeracil + oteracil potassium; OX, oxaliplatin; CAP, capecitabine; IRI, irinotecan; Mayo, 5-FU + isovorin; MIFL, 5-FU + LV + irinotecan; 5-FU, 5-fluorouracil.

was used. The NOS is composed of eight items that assess patient selection, study, comparability and outcome (42). A summary of the quality assessment results is demonstrated in Table I.

\section{Data analysis}

$O S$. The meta-analysis was performed on 10 studies (887 patients) investigating the association between TP and OS. As the heterogeneity test was not significant $\left(\chi^{2}=11.42\right.$; $\mathrm{P}=0.326 ; \mathrm{I}^{2}=12.4 \%$ ), the fixed-effects model was used to calculate the HR. The pooled HR from the 10 studies was 0.76 ( $\mathrm{P}=0.031$; 95\% CI, 0.59-0.98; Fig. 2), which indicated that there was significant correlation between the OS and TP in CRC patients treated with 5-FU-based chemotherapy. The funnel plot and Egger's test demonstrated that no significant publication bias was detected $(\mathrm{P}=0.963$; Fig. 3$)$. In order to test the stability of the result, the studies whose quality score was below five and was displayed as significant were excluded. Sensitivity analysis demonstrated that the result of OS was stable (Fig. 4).

Following this, analysis was restricted to the five studies assessing TP expression in primary tumors. The pooled HR was $0.62(\mathrm{P}=0.005 ; 95 \% \mathrm{CI}, 0.45-0.87)$ without evidence of study heterogeneity $\left(\chi^{2}=1.20 ; \mathrm{P}=0.878 ; \mathrm{I}^{2}=0.0 \%\right)$. Five studies assessed TP expression in metastatic tumors, and the pooled HR was $0.90(\mathrm{P}=0.594 ; 95 \% \mathrm{CI}, 0.61-1.32)$, without evidence of heterogeneity $\left(\chi^{2}=2.85 ; \mathrm{P}=0.583 ; \mathrm{I}^{2}=0.0 \%\right.$;
Fig. 5). To assess the effect of the method used to evaluate TP expression, subgroup analysis was performed based on IHC or qPCR. HR was pooled from all 10 studies using either qPCR or IHC. A larger pooled HR was demonstrated in studies using the qPCR method $(\mathrm{HR}=0.87 ; \mathrm{P}=0.396 ; 95 \%$ CI, 0.62-1.21), compared with that from studies using the IHC method $(\mathrm{HR}=0.58 ; \mathrm{P}=0.022 ; 95 \% \mathrm{CI}, 0.40-0.85)$. There was no evidence of heterogeneity in qPCR-based studies $\left(\chi^{2}=2.65\right.$; $\left.\mathrm{P}=0.619 ; \mathrm{I}^{2}=0.0 \%\right)$ or IHC-based studies $\left(\chi^{2}=7.50 ; \mathrm{P}=0.900\right.$; $\mathrm{I}^{2}=0.0 \%$; Fig. 6 ).

ORR, PFS, DFS and RFS. Table III detailed the meta-analysis results of ORR, PFS, DFS and RFS. Of the 15 eligible studies, five $(25,32-34,36)$ (200 patients) reported data available for ORR, and the pooled OR was $0.822(\mathrm{P}=0.628 ; 95 \% \mathrm{CI}$, $0.373-1.812)$ with evidence of heterogeneity $\left(\chi^{2}=10.56 ; \mathrm{P}=0.031\right.$; $\mathrm{I}^{2}=62.4 \%$ ). The Egger's test demonstrated that no publication bias was detected $(\mathrm{P}=0.096)$. There were three studies $(4,29,33)$ for PFS, three $(26,27,30)$ for DFS and two $(3,31)$ for RFS. The pooled HRs for PFS, DFS and RFS were $0.752(\mathrm{P}=0.511 ; 95 \%$ CI, 0.321-1.760), $1.415(\mathrm{P}=0.579 ; 95 \% \mathrm{CI}, 0.416-4.816)$ and 0.711 ( $\mathrm{P}=0.022 ; 95 \% \mathrm{CI}, 0.531-0.951)$ respectively, all without evidence of heterogeneity. The number of studies used here is not large enough to reach a conclusion. Therefore, more trials regarding this should be performed to fully determine the association between TP and survival in patients with CRC treated with 5-FU-based chemotherapy. 
Table II. Summary of TP expression for individual studies.

\begin{tabular}{|c|c|c|c|c|c|c|c|}
\hline $\begin{array}{l}\text { Author/(Refs.), } \\
\text { year }\end{array}$ & $\begin{array}{l}\text { Treatment } \\
\text { setting }\end{array}$ & $\begin{array}{l}\text { Median } \\
\text { follow-up } \\
\text { period, } \\
\text { months }\end{array}$ & $\begin{array}{c}\text { Association } \\
\text { with } \\
\text { prognosis }\end{array}$ & $\begin{array}{l}\text { Lesion } \\
\text { tested }\end{array}$ & $\begin{array}{c}\text { TP } \\
\text { evaluation } \\
\text { method }\end{array}$ & $\begin{array}{c}\text { Study } \\
\text { size } \\
\text { (no. of } \\
\text { patients) }\end{array}$ & $\begin{array}{c}\text { Patients } \\
\text { with high } \\
\text { TP level, } \\
\text { n (\%) }\end{array}$ \\
\hline Ahn et al (24), 2005 & Metastatic & NS & None & Primary & $\mathrm{IHC}$ & 45 & $22(49)$ \\
\hline Kataoka et al (25), 2015 & Metastatic & 42.6 & None & Both & qPCR & 36 & $18(50)$ \\
\hline Shigeta et al (4), 2014 & Primary & 66 & Good & Primary & qPCR & 101 & $71(70)$ \\
\hline Ogawa et al (35), 2014 & Primary & 12 & Good & Primary & RT-qPCR & 54 & $27(50)$ \\
\hline Donada et al (36), 2011 & Primary & 91.2 & None & Primary & qPCR & 55 & $27(49)$ \\
\hline Petrioli et al (26), 2010 & Metastatic & 20.4 & Good & Metastatic & $\mathrm{IHC}$ & 41 & $21(51)$ \\
\hline Lindskog et al (5), 2014 & Metastatic & 29 & Poor & Primary & qPCR & 125 & $62(50)$ \\
\hline Yamada et al (27), 2008 & Primary & 30 & None & Primary & qPCR & 103 & $51(50)$ \\
\hline Jensen et al (28), 2008 & Both & NS & None & Primary & $\mathrm{IHC}$ & 300 & $150(50)$ \\
\hline Yanagisawa et al (37), 2007 & Primary & 15.7 & None & Primary & $\mathrm{IHC}$ & 13 & $5(39)$ \\
\hline Meropol et al (29), 2006 & Metastatic & NS & Good & Both & $\mathrm{IHC}$ & 67 & $24(36)$ \\
\hline Ichikawa et al (38), 2003 & Metastatic & 14 & None & Primary & qPCR & 37 & $18(49)$ \\
\hline Tokunaga et al (39), 2002 & Both & NS & Poor & NS & $\mathrm{IHC}$ & 80 & $54(68)$ \\
\hline Metzger et al (40), 1998 & Metastatic & NS & Poor & NS & RT-qPCR & 38 & $10(26)$ \\
\hline Soong et al (41), 2008 & Primary & 52.4 & Poor & Primary & $\mathrm{IHC}$ & 130 & $86(66)$ \\
\hline
\end{tabular}

TP, thymidine phosphorylase; qPCR, quantitative polymerase chain reaction; RT-qPCR, reverse transcription-quantitative polymerase chain reaction; IHC, immunohistochemistry; NS, not stated.

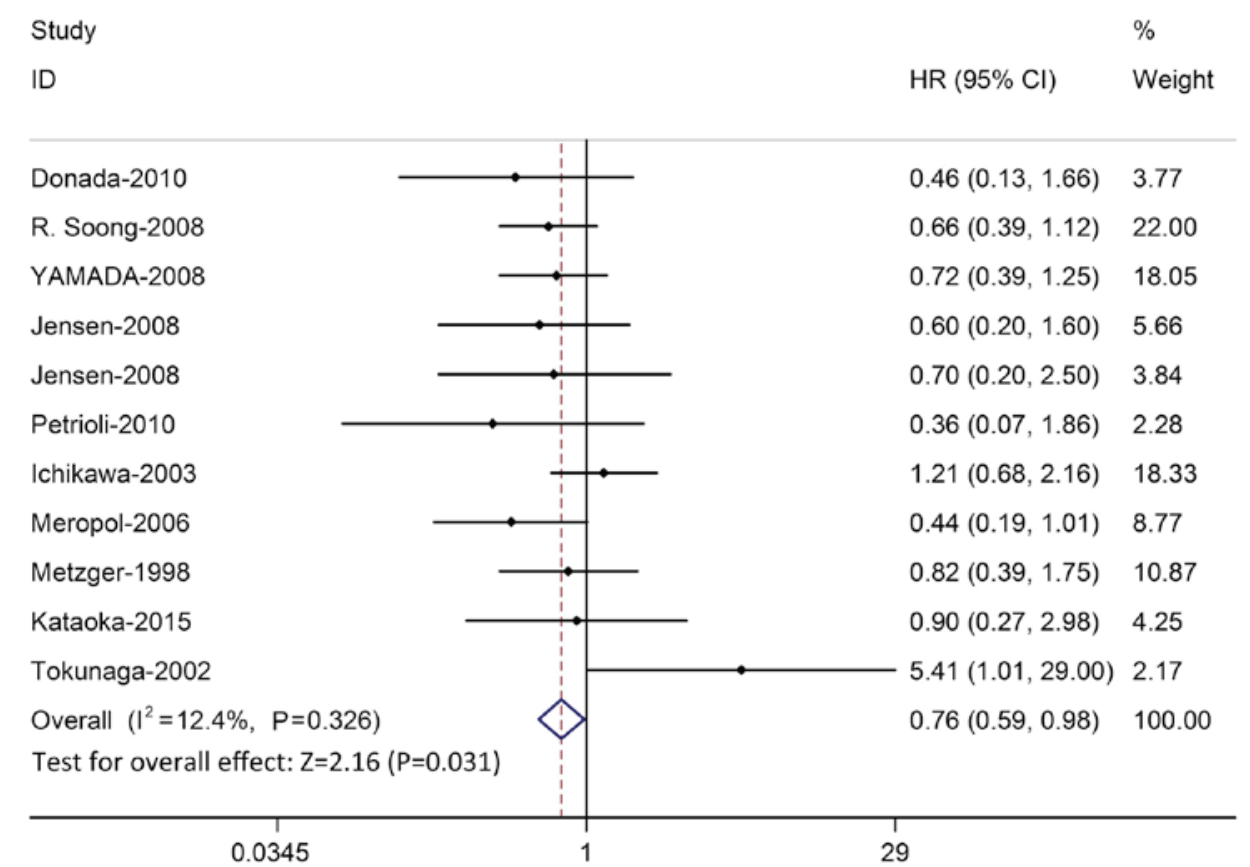

Figure 2. Forest plot of HR for the association of TP expression with overall survival ( $\mathrm{TP}^{+} /$high vs. $\mathrm{TP}^{-} /$low). $\mathrm{HR}$, hazard ratio; $\mathrm{TP}$, thymidine phosphorylase; CI, confidence interval.

\section{Discussion}

The present meta-analysis reviewed 15 eligible articles to determine the association between TP expression and the prognosis of patients with CRC treated with 5-FU-based chemotherapy. The results demonstrated that higher TP expression is correlated with better prognosis, as evaluated by OS, and may serve as a predictor of prognosis in 5-FU-based 
Table III. Results of meta-analysis for ORR, PFS, DFS and RFS.

\begin{tabular}{lcccccccc}
\hline & & & & & \multicolumn{3}{c}{ Heterogeneity } \\
\cline { 5 - 7 } Index & $\begin{array}{c}\text { No. } \\
\text { of articles }\end{array}$ & $\begin{array}{c}\text { Pooled odds } \\
\text { ratio/hazard ratio }\end{array}$ & $\begin{array}{c}95 \% \text { confidence } \\
\text { interval }\end{array}$ & P-value & $\chi^{2}$ & $\mathrm{I}^{2}$ & $\mathrm{P}^{1}$ & $\mathrm{P}^{2}$ \\
\hline ORR & 5 & 0.822 & $0.373-1.812$ & 0.628 & 10.56 & $62.4 \%$ & 0.031 & 0.096 \\
PFS & 3 & 0.752 & $0.321-1.760$ & 0.511 & 13.49 & $85.2 \%$ & 0.0001 & 0.361 \\
DFS & 3 & 1.415 & $0.416-4.816$ & 0.579 & 5.14 & $61.1 \%$ & 0.077 & 0.586 \\
RFS & 2 & 0.711 & $0.531-0.951$ & 0.022 & 0.10 & $0.00 \%$ & 0.754 & - \\
\hline
\end{tabular}

ORR, objective response rate; PFS, progression-free survival; DFS, disease-free survival; RFS, relapse-free survival; $\mathrm{P}^{1}$, $\mathrm{P}$-value of heterogeneity; $\mathrm{P}^{2}, \mathrm{P}$-value of Egger's test; -, two studies is too few to perform Egger's test.

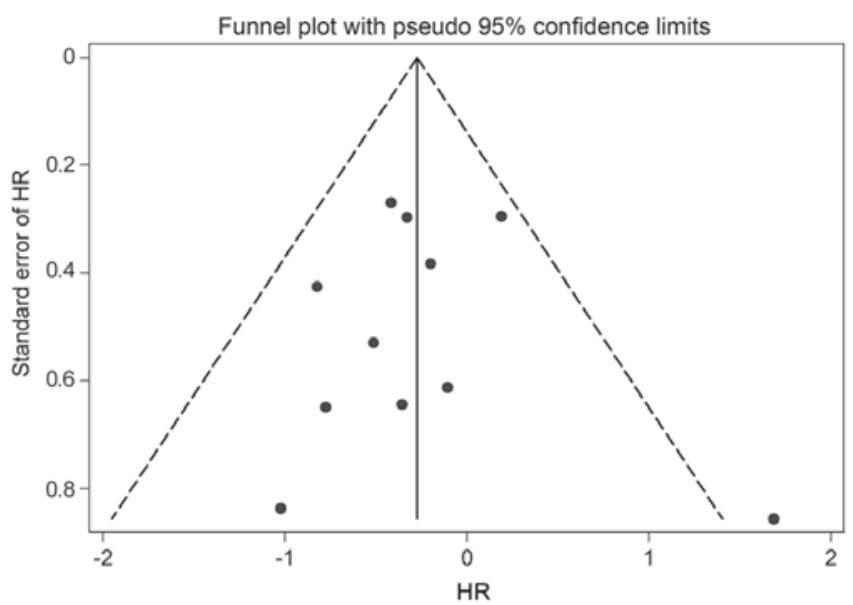

Figure 3. Funnel plot of overall survival. HR, hazard ratio.

chemotherapy for CRC. However, this is not the case for ORR, PFS, DFS and RFS. Furthermore, this seems to be the case for patients with primary tumors and patients whose TP evaluation method is IHC, according to subgroup analysis of OS. In patients with metastatic tumors and patients whose TP evaluation method is qPCR, TP expression does not appear to predict prognosis.

The value of high TP expression in predicting good OS appears to be stronger in studies conducted in a primary treatment setting than those conducted in a metastatic treatment setting. For studies that were all in a metastatic treatment setting and reported ORR and PFS, there was no significant difference between TP expression and prognosis of CRC. This may partly result from different 5-FU-based chemotherapy regimens that primary and metastatic tumors are usually treated with. Different drugs, such as oxaplatin, irinotecan and capecitabine, accompanied with 5-FU regimens may cause various effects (43). For DFS and RFS, which were both conducted in primary treatment settings, the opposite conclusion was reached. TP expression does not appear to predict prognosis of CRC treated with 5-FU-based chemotherapy in DFS; however, it does predict a good prognosis in RFS, although, there were only three studies dealing with DFS and two dealing with RFS. Therefore, these results should be interpreted with caution considering the small number of contributing studies.
Furthermore, it was observed that higher TP expression may predict better prognosis in studies using IHC but not qPCR. This may be due to the different cut-off values used to assign TP status in the qPCR studies. Dichotomization in some of the qPCR studies depended on median value, while others depended on likely response. This was not the case for the IHC studies. TP expression in the IHC studies was quantified by a visual grading system based on the intensity of staining and classified into four grades, from 0 (undetectable staining) to 3 (very high intensity of staining).

As TP is an enzyme that not only participates in 5-FU metabolism, but also converts 5'-DFUR to 5-FU (21), it was hypothesized to be a potential predictor of response. However, experimental studies also reported that high TP expression is associated with the decreased sensitivity of CRC to 5-FU (44) and some clinical trials demonstrated no clinically useful correlation between TP expression and the response to post-operative adjuvant chemotherapy with agents such as 5-FU/leucovorin and 5'-DFUR (28). The earlier results were always controversial, while the results of the present meta-analysis are consistent with the previous three articles by Ogawa et al (35), Petrioli et al (26) and Meropol et al (29), which indicated a positive correlation between high TP expression and positive outcomes in CRC treated with 5-FU-based chemotherapy.

The results of the association between TP expression and the prognosis of 5-FU-based chemotherapy in CRC varied among the 15 articles chosen for analysis. Six articles by Ahn et al (24), Yamada et al (27), Jensen et al (28), Donada et al (36) and Ichikawa et al (38) indicated that there was no association between the expression of TP and the prognosis of 5-FU-based chemotherapy. Two articles by Kataoka et al (25) and Yanagisawa et al (37) identified the association but did not express it in detail. However, the trial conducted by Shigeta et al (4) indicated that high TP expression was associated with a trend for improved prognosis in RFS. There were also two articles by Lindskog et al (5) and Tokunaga et al (39) that indicated that high or low TP expression was an independent poor prognostic factor, in contrast to the present result. The apparent discrepancy may be explained in several ways. First, sample size may be insufficient to achieve adequate statistical power for specific biomarker end points. The number of CRC patients participating in the trials should be higher, so that the discrepancy 
Study

ID
$\%$

$\mathrm{HR}(95 \% \mathrm{Cl}) \quad$ Weight

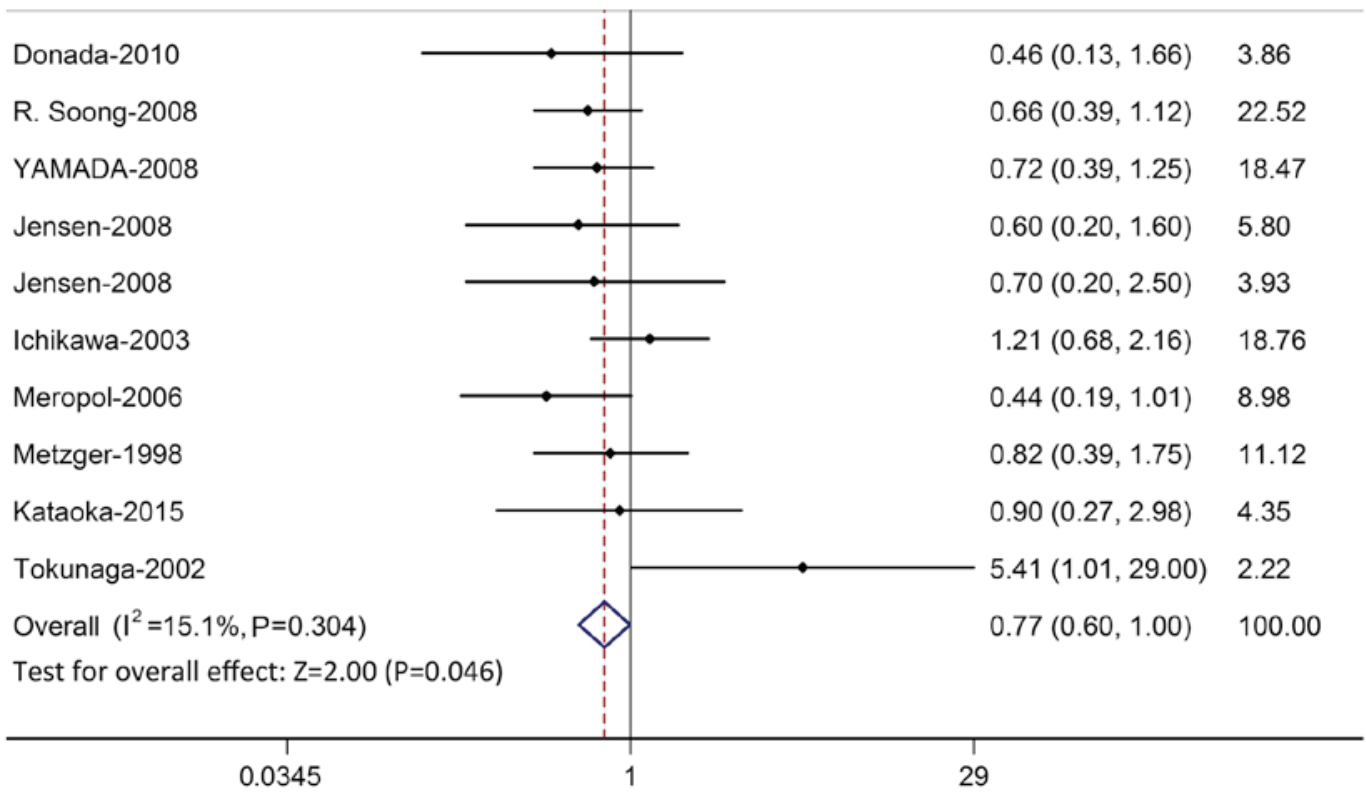

Figure 4. Forest plot of sensitivity analysis for overall survival. HR, hazard ratio; CI, confidence interval.

Study

ID
$\%$

$\mathrm{HR}(95 \% \mathrm{Cl}) \quad$ Weight

\section{Primary}

Donada-2010

R. Soong-2008

YAMADA-2008

Jensen-2008

Meropol-2006

Subtotal $\left(I^{2}=0.0 \%, P=0.878\right)$

Test for overall effect: $Z=2.80(P=0.005)$

Metastatic

Jensen-2008

Petrioli-2010

Ichikawa-2003

Metzger-1998

Kataoka-2015

Subtotal $\left(I^{2}=0.0 \%, P=0.583\right)$

Test for overall effect: $Z=0.53$ ( $P=0.594)$

Heterogeneity between groups: $P=0.156$

Overall $\left(I^{2}=0.0 \%, P=0.734\right)$

Test for overall effect: $Z=2.48(P=0.013)$
$0.46(0.13,1.66) 3.86$

$0.66(0.39,1.12) 22.49$

$0.72(0.39,1.25) 18.45$

$0.70(0.20,2.50) 3.92$

$0.44(0.19,1.01) 8.97$

$0.62(0.45,0.87) 57.70$

$0.60(0.20,1.60) 5.79$

$0.36(0.07,1.86) 2.33$

$1.21(0.68,2.16) 18.74$

$0.82(0.39,1.75) 11.11$

$0.90(0.27,2.98) 4.34$

$0.90(0.61,1.32) 42.30$

$0.73(0.57,0.94) 100.00$

$0.07 \quad 14.3$

Figure 5. Forest plot of HR for the association of TP expression with overall survival based on treatment setting (TP $/$ high vs. TP/low). HR, hazard ratio; TP, thymidine phosphorylase; CI, confidence interval.

between results may be minimized. Second, the inverse association between TP expression and the DFS and response to 5-FU may be a consequence of the role of TP as an angiogenetic factor. TP, which is identical to platelet-derived endothelial cell growth factor (45), and the degeneration products, thymine and 2-deoxy-D-ribose, have angiogenic 


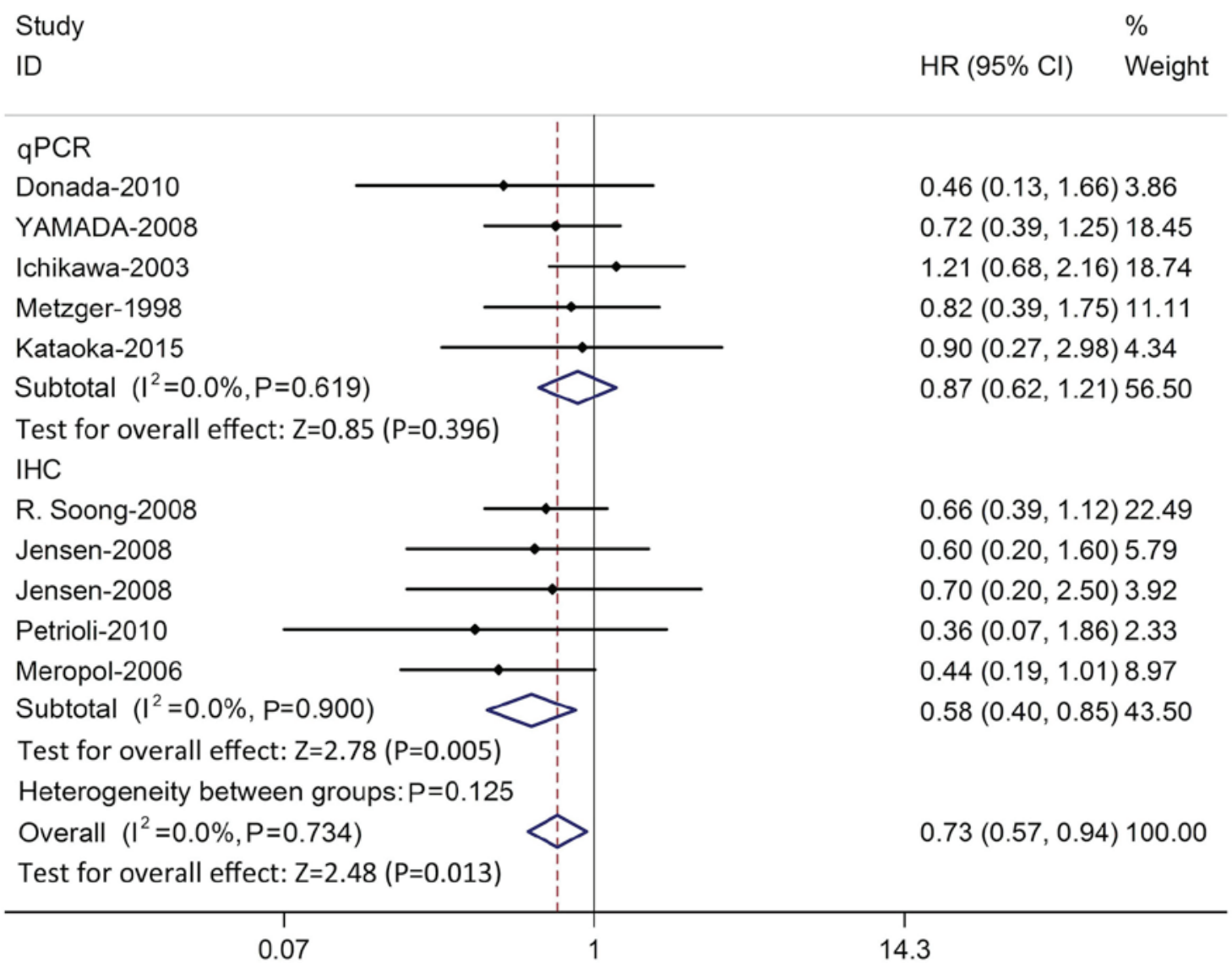

Figure 6. Forest plot of $\mathrm{HR}$ for the association of $\mathrm{TP}$ expression with overall survival based on TP evaluation method (TP+/high vs. $\left.\mathrm{TP}^{-} / \mathrm{low}\right)$. $\mathrm{HR}$, hazard ratio; $\mathrm{TP}$, thymidine phosphorylase; CI, confidence interval; qPCR, quantitative polymerase chain reaction; IHC, immunohistochemistry.

and anti-apoptotic effects (46). Although the role of TP in tumor proliferation is yet to be fully elucidated, TP has angiogenetic activity and its enzymatic activity is required for angiogenesis (47). A previous study demonstrated that TP prevents hypoxia-induced apoptosis and that the degradation products of thymidine are involved in this response (48). Thus, TP expression may provide an advantage for tumor growth in CRC by not only increasing the intratumoral microvessel density, but also by attenuating apoptosis (46), which suggests that the suppression of TP may result in the inhibition of growth of TP-positive tumors in patients with CRC.

Furthermore, the present results suggested that the association between TP expression and prognosis of CRC is different between primary CRC tumors and metastatic CRC tumors. It was hypothesized that TP may be correlated with metastasis and advanced CRC, and it was also considered that TP may induce angiogenesis in tumor tissues. Perhaps one of the dual roles of TP, that it participates in the metabolism of 5-FU in CRC cancer cells to defend cancer cells, is stronger than the role of it inducing angiogenesis. However, the mechanisms need to be further studied, as the interactions between TP expression and other factors of angiogenesis are not known.

The present meta-analysis has several notable limitations. First, the cut-off line of high and low TP expression was different across each trial, and it was not defined with a standardized value in the present review. Second, only six trials reported HRs and variances, and so HRs and variances had to be calculated or converted for other trials from the reported survival curves, which may introduce unavoidable bias. Third, the majority of trials were retrospective trials, which may cause selective bias. Fourth, the lesions tested were predominantly from primary tumors, which may result in bias. Finally, 5-FU, utilized as a first line treatment for CRC, is used as an intramuscular injection agent, or in combination with oral drugs, such as capecitabine and tegafur, which may influence the efficacy of 5-FU.

In spite of the above limitations, the present meta-analysis demonstrated that higher TP expression is correlated with better prognosis in CRC treated with 5-FU-based chemotherapy. Additional investigation is necessary to provide more specific information about the association between TP expression and 5-FU-based treatment for patients with CRC.

\section{Acknowledgements}

The present sudy was supported by Guangdong Provincial Department of Science and Technology (grant no. 2014B020212016), Guangdong Innovative Research Team Program (grant no. 2009010058), National Key Clinical Discipline and Overseas Excellent Professor Project, Ministry of Education, China. 


\section{References}

1. Siegel RL, Miller KD and Jemal A: Cancer Statistics, 2017. CA Cancer J Clin 67: 7-30, 2017.

2. Torre LA, Bray F, Siegel RL, Ferlay J, Lortet-Tieulent J and Jemal A: Global cancer statistics, 2012. CA Cancer J Clin 65: $87-108,2015$

3. de Gramont A, Figer A, Seymour M, Homerin M, Hmissi A, Cassidy J, Boni C, Cortes-Funes H, Cervantes A, Freyer G, et al: Leucovorin and fluorouracil with or without oxaliplatin as first-line treatment in advanced colorectal cancer. J Clin Oncol 18: 2938-2947, 2000.

4. Shigeta K, Ishii Y, Hasegawa H, Okabayashi K and Kitagawa Y: Evaluation of 5-fluorouracil metabolic enzymes as predictors of response to adjuvant chemotherapy outcomes in patients with stage II/III colorectal cancer: A decision-curve analysis. World J Surg 38: 3248-3256, 2014.

5. Lindskog EB, Derwinger K, Gustavsson B, Falk P and Wettergren Y: Thymidine phosphorylase expression is associated with time to progression in patients with metastatic colorectal cancer. BMC Clin Pathol doi 14: 25, 2014.

6. Mayer RJ: Moving beyond fluorouracil for colorectal cancer. N Engl J Med 343: 963-964, 2000

7. Lee JJ, Beumer JH and Chu E: Therapeutic drug monitoring of 5-fluorouracil. Cancer Chemother Pharmacol 78: 447-464, 2016.

8. André T, Boni C, Mounedji-Boudiaf L, Navarro M, Tabernero J, Hickish T, Topham C, Zaninelli M, Clingan P, Bridgewater J, et al: Oxaliplatin, fluorouracil and leucovorin as adjuvant treatment for colon cancer. N Engl J Med 350: 2343-2351, 2004.

9. Maindrault-Goebel F, Louvet C, André T, Carola E, Lotz JP, Molitor JL, Garcia ML, Gilles-Amar V, Izrael V, Krulik M and de Gramont A: Oxaliplatin added to the simplified bimonthly leucovorin and 5-fluorouracil regimen as second-line therapy for metastatic colorectal cancer (FOLFOX6). GERCOR. Eur J Cancer 35: 1338-1342, 1999.

10. Quasar Collaborative Group Gray R, Barnwell J, McConkey C, Hills RK, Williams NS and Kerr DJ: Adjuvant chemotherapy versus observation in patients with colorectal cancer: A randomized study. Lancet 370: 2020-2029, 2007.

11. Twelves C, Wong A, Nowacki MP, Abt M, Burris H III, Carrato A, Cassidy J, Cervantes A, Fagerberg J, Georgoulias V, et al: Capecitabine as adjuvant treatment for stage III colon cancer. N Engl J Med 352: 2696-2704, 2005.

12. Lembersky BC, Wieand HS, Petrelli NJ, O'Connell MJ, Colangelo LH, Smith RE, Seay TE, Giguere JK, Marshall ME, Jacobs AD, et al: Oral uracil and tegafur plus leucovorin compared with intravenous fluorouracil and leucovorin in stage II and III carcinoma of the colon: Results from National Surgical Adjuvant Breast and Bowel Project Protocol C-06. J Clin Oncol 24: 2059-2064, 2006

13. Labianca R, Nordlinger B, Beretta GD, Brouquet A and Cervantes A; ESMO Guidelines Working Group: Primary colon cancer: ESMO Clinical Practice Guidelines for diagnosis, adjuvant treatment and follow-up. Ann Oncol 21 (Suppl 5): v70-v77, 2010.

14. Deboever G, Hiltrop N, Cool M and Lambrecht G: Alternative treatment options in colorectal cancer patients with 5-fluorouracil- or capecitabine-induced cardiotoxicity. Clin Colorectal Cancer 12: 8-14, 2013.

15. Bronckaers A, Gago F, Balzarini J and Liekens S: The dual role of thymidine phosphorylase in cancer development and chemotherapy. Med Res Rev 29: 903-953, 2009.

16. O'Brien TS, Fox SB, Dickinson AJ, Turley H, Westwood M, Moghaddam A, Gatter KC, Bicknell R and Harris AL: Expression of the angiogenic factor thymidine phosphorylase/platelet-derived endothelial cell growth factor in primary bladder cancers. Cancer Res 56: 4799-4804, 1996.

17. Miwa M, Ura M, Nishida M, Sawada N, Ishikawa T, Mori K, Shimma N, Umeda I and Ishitsuka H: Design of a novel oral fluoropyrimidine carbamate, capecitabine, which generates 5 -fluorouracil selectively in tumours by enzymes concentrated in human liver and cancer tissue. Eur J Cancer 34: 1274-1281, 1998

18. Takebayashi Y, Yamada K, Miyadera K, Sumizawa T, Furukawa T, Kinoshita F, Aoki D, Okumura H, Yamada Y, Akiyama S and Aikou T: The activity and expression of thymidine phosphorylase in human solid tumours. Eur J Cancer 32A: 1227-1232, 1996.

19. Nozawa T, Enomoto T, Koshida Y, Sato Y and Kuranami M Specific enhanced expression of platelet-derived endothelial cell growth factor in submucosa of human colorectal cancer. Dis Colon Rectum 47: 2093-2100, 2004
20. Amatori F, Di Paolo A, Del Tacca M, Fontanini G, Vannozzi F, Boldrini L, Bocci G, Lastella M and Danesi R: Thymidylate synthase, dihydropyrimidine dehydrogenase and thymidine phosphorylase expression in colorectal cancer and normal mucosa in patients. Pharmacogenet Genomics 16: 809-816, 2006

21. de Bruin M, van Capel T, Van der Born K, KruytFA, Fukushima M, Hoekman K, Pinedo HM and Peters GJ: Role of platelet-derived endothelial cell growth factor/thymidine phosphorylase in fluoropyrimidine sensitivity. Br J Cancer 88: 957-64, 2003.

22. Moghaddam A, Zhang HT, Fan TP, Hu DE, Lees VC, Turley H, Fox SB, Gatter KC, Harris AL and Bicknell R: Thymidine phosphorylase is angiogenic and promotes tumor growth. Proc Natl Acad Sci USA 92: 998-1002, 1995.

23. Takebayashi Y, Akiyama S, Akiba S, Yamada K, Miyadera K, Sumizawa T, Yamada Y, Murata F and Aikou T: Clinicopathologic and prognostic significance of an angiogenic factor, thymidine phosphorylase, in human colorectal carcinoma. J Natl Cancer Inst 88: 1110-1117, 1996.

24. Ahn MJ, Choi JH, Oh HS, Lee YY, Kim IS, Choi IY, Lee KH, Song KW and Park CK: Thymidylate synthase, thymidine phosphorylase, VEGF and p53 protein expression in primary colorectal cancer for predicting response to 5-fluorouracil-based chemotherapy. Cancer Res Treat 37: 216-222, 2005.

25. Kataoka K, Kanazawa A, Nakajima A, Yamaguchi A and Arimoto A: Prognostic value of biomarkers in metastatic colorectal cancer patients. J Surg Res 194: 343-350, 2015.

26. Petrioli R, Bargagli G, Lazzi S, Pascucci A, Francini E, Bellan C, Conca R, Martellucci I, Fiaschi AI, Lorenzi B, et al: Thymidine phosphorylase expression in metastatic sites is predictive for response in patients with colorectal cancer treated with continuous oral capecitabine and biweekly oxaliplatin. Anticancer Drugs 21: 313-319, 2010.

27. Yamada $H$, Iinuma $H$ and Watanabe T: Prognostic value of 5-fluorouracil metabolic enzyme genes in Dukes' stage B and C colorectal cancer patients treated with oral 5-fluorouracil-based adjuvant chemotherapy. Oncol Rep 19: 729-735, 2008.

28. Jensen SA, Vainer B, Witton CJ, Jørgensen JT and Sørensen JB: Prognostic significance of numeric aberrations of genes for thymidylate synthase, thymidine phosphorylase and dihydrofolate reductase in colorectal cancer. Acta Oncol 47: 1054-1061, 2008.

29. Meropol NJ, Gold PJ, Diasio RB, Andria M, Dhami M, Godfrey T, Kovatich AJ, Lund KA, Mitchell E and Schwarting R: Thymidine phosphorylase expression is associated with response to capecitabine plus irinotecan in patients with metastatic colorectal cancer. J Clin Oncol 24: 4069-4077, 2006

30. Tierney JF, Stewart LA, Ghersi D, Burdett S and Sydes MR: Practical methods for incorporating summary time-to-event data into meta-analysis. Trials 8: 16, 2007.

31. Parmar MK, Torri V and Stewart L: Extracting summary statistics to perform meta-analyses of the published literature for survival endpoints. Stat Med 17: 2815-2834, 1998.

32. Duffaud F and Therasse P: New guidelines to evaluate the response to treatment in solid tumors. Bull Cancer 87: 881-886, 2000 (In French).

33. Miller AB, Hoogstraten B, Staquet M and Winkler A: Reporting results of cancer treatment. Cancer 47: 207-214, 1981.

34. Egger M, Davey Smith G, Schneider M and Minder C: Bias in meta-analysis detected by a simple, graphical test. BMJ 315: 629-634, 1997.

35. Ogawa M, Watanabe M, Mitsuyama Y, Anan T, Ohkuma M, Kobayashi T, Eto K and Yanaga K: Thymidine phosphorylase mRNA expression may be a predictor of response to post-operative adjuvant chemotherapy with S-1 in patients with stage III colorectal cancer. Oncol Lett 8: 2463-2468, 2014.

36. Donada M, Bonin S, Nardon E, De Pellegrin A, Decorti G and Stanta G: Thymidilate synthase expression predicts longer survival in patients with stage II colon cancer treated with 5 -flurouracil independently of microsatellite instability. J Cancer Res Clin Oncol 137: 201-210, 2011

37. Yanagisawa $Y$, Maruta F, Iinuma N, Ishizone S, Koide $N$, Nakayama J and Miyagawa S: Modified Irinotecan/5FU/ Leucovorin therapy in advanced colorectal cancer and predicting therapeutic efficacy by expression of tumor-related enzymes. Scand J Gastroenterol 42: 477-484, 2007.

38. Ichikawa W, Uetake H, Shirota Y, Yamada H, Takahashi $T$, Nihei Z, Sugihara K, Sasaki Y and Hirayama R: Both gene expression for orotate phosphoribosyltransferase and its ratio to dihydropyrimidine dehydrogenase influence outcome following fluoropyrimidine-based chemotherapy for metastatic colorectal cancer. Br J Cancer 89: 1486-1492, 2003. 
39. Tokunaga Y, Hosogi H, Hoppou T, Nakagami M, Tokuka A and Ohsumi K: Prognostic value of thymidine phosphorylase/platelet-derived endothelial cell growth factor in advanced colorectal cancer after surgery: Evaluation with a new monoclonal antibody. Surgery 131: 541-547, 2002.

40. Metzger R, Danenberg K, Leichman CG, Salonga D, Schwartz EL, Wadler S, Lenz HJ, Groshen S, Leichman L and Danenberg PV: High basal level gene expression of thymidine phosphorylase (platelet-derived endothelial cell growth factor) in colorectal tumors is associated with nonresponse to 5-fluorouracil. Clin Cancer Res 4: 2371-2376, 1998.

41. Soong R, Shah N, Salto-Tellez M, Tai BC, Soo RA, Han HC, Ng SS, Tan WL, Zeps N, Joseph D, et al: Prognostic significance of thymidylate synthase, dihydropyrimidine dehydrogenase and thymidine phosphorylase protein expression in colorectal cancer patients treated with or without 5-fluorouracil-based chemotherapy. Ann Oncol 19: 915-919, 2008.

42. Stang A: Critical evaluation of the Newcastle-Ottawa scale for the assessment of the quality of nonrandomized studies in meta-analyses. Eur J Epidemiol 25: 603-605, 2010.

43. Tournigand C, André T, Achille E, Lledo G, Flesh M, MeryMignard D, Quinaux E, Couteau C, Buyse M, Ganem G, et al: FOLFIRI followed by FOLFOX6 or the reverse sequence in advanced colorectal cancer: A randomized GERCOR study. J Clin Oncol 22: 229-237, 2004.
44. Salonga D, Danenberg KD, Johnson M, Metzger R, Groshen S, Tsao-Wei DD, Lenz HJ, Leichman CG, Leichman L, Diasio RB and Danenberg PV: Colorectal tumors responding to 5-fluorouracil have low gene expression levels of dihydropyrimidine dehydrogenase, thymidylate synthase, and thymidine phosphorylase. Clin Cancer Res 6: 1322-1327, 2000.

45. Nishimura G, Terada I, Kobayashi T, Ninomiya I, Kitagawa H, Fushida S, Fujimura T, Kayahara M, Shimizu K, Ohta T and Miwa K: Thymidine phosphorylase and dihydropyrimidine dehydrogenase levels in primary colorectal cancer show a relationship to clinical effects of 5'-deoxy-5-fluorouridine as adjuvant chemotherapy. Oncol Rep 9: 479-482, 2002.

46. Matsuura T, Kuratate I, Teramachi K, Osaki M, Fukuda Y and Ito $\mathrm{H}$ : Thymidine phosphorylase expression is associated with both increase of intratumoral microvessels and decrease of apoptosis in human colorectal carcinomas. Cancer Res 59: 5037-5040, 1999.

47. Elamin YY, Rafee S, Osman N, O Byrne KJ and Gately K: Thymidine phosphorylase in cancer; enemy or friend? Cancer Microenviron 9: 33-43, 2016.

48. Bijnsdorp IV, Azijli K, Jansen EE, Wamelink MM, Jakobs C, Struys EA, Fukushima M, Kruyt FA and Peters GJ: Accumulation of thymidine-derived sugars in thymidine phosphorylase overexpressing cells. Biochem Pharmacol 80: 786-792, 2010. 